\title{
Photon and Elementary Particles Theory
}

\author{
Xiangyao $\mathrm{Wu}^{1}$, Benshan $\mathrm{Wu}^{1}$, Siqi Zhang1, Xiaojing Liu${ }^{1}$, Yiheng $\mathrm{Wu}^{2}$, Jiping Liu${ }^{1}$, Xiaoru Zhang1, \\ Han Liu' ${ }^{1}$ Ji Ma ${ }^{1}$, Hong Li ${ }^{1}$, Li Xiao' ${ }^{1}$ Yuntuan Fang ${ }^{3}$ \\ ${ }^{1}$ Institute of Physics, Jilin Normal University, Siping, China \\ ${ }^{2}$ College of Physics and Electrical Engineering, Anqing Normal University, Anqing, China \\ ${ }^{3}$ School of Computer Science and Telecommunication Engineering, Jiangsu University, Zhenjiang, China \\ Email: *wuxy2066@163.com
}

\begin{abstract}
How to cite this paper: $\mathrm{Wu}, \mathrm{X}$.Y., Wu, B.S., Zhang, S.Q., Liu, X.J., Wu, Y.H., Liu, J.P., Zhang, X.R., Liu, H., Ma, J., Li, H., Xiao, L. and Fang, Y.T. (2017) Photon and Elementary Particles Theory. Journal of Modern Physics, 8, 1850-1867.

https://doi.org/10.4236/jmp.2017.811109
\end{abstract}

Received: June 25, 2017

Accepted: October 21, 2017

Published: October 24, 2017

Copyright $\odot 2017$ by authors and Scientific Research Publishing Inc. This work is licensed under the Creative Commons Attribution International License (CC BY 4.0).

http://creativecommons.org/licenses/by/4.0/

\begin{abstract}
At present, the research of single-photon is a hot topic, it has been widely applied in quantum measurement, quantum entanglement and quantum information. In this paper, we have proposed a new single photon theory, which is the vector potential $\boldsymbol{A}$ rotation at the vertical motion direction of photon, it can produce the microscopic electric field and magnetic field, and they satisfy the Maxwell equations. We have calculated photon spin, momentum, energy, and found there are left-handed and right-handed photon. In addition, we study the multi-photon theory and the dynamics theory between photons interaction. On this basis, we have further given the compound structures of electron, proton, neutron, neutrino, $\pi$ meson, dark matter with the photon structural theory, and calculated their mass, charge and spin.
\end{abstract}

\section{Keywords}

Photon, Vector-Potential Rotation, Elementary Particles, Dark Matter

\section{Introduction}

The wave theory of light was put forward by Huyghens and was later proved by interference and diffraction experiments, which became the greatest achievements of the $19^{\text {th }}$ century. In order to explain the spectrum of black-body radiation, Planck postulated the process of emission and absorption of radiation by atoms occurs discontinuously in quanta, i.e., the emission of black-body was energy quantization with value of $\hbar \omega$ [1]. In 1902, Lenard discovered that energy of electrons in photoelectric effect does not depend on the intensity of light, while it depends on the light frequency [2]. In 1905, Einstein pointed out that the discovery of Lenard meant that energy of light is in a form of localized light quanta [3]. The proof that Einstein light quanta behave as particles was given by 
the Compton scattering experiment of X-rays on electrons in 1923 [4].

There are still many puzzling aspects about light nature. A central point is the distinction between particle and field viewpoint of light. In the old days, this was called wave-particle duality. In quantum electrodynamics, we treat photon as the quanta of the classical electromagnetic field quantization. A key question is, we view light as being comprised of particles called photons, or one view light as a field [5]. In 1951, Einstein complained that after fifty years of conscious meditation he had not come any closer to the answer to the question what are light quanta. He added: "today any brute believes that he knows the answer, but he is wrong" [6].

In the last three decades, the research of single-photon is a hot and widely discussed topic in the physics community, it has been applied in quantum measurement [7] [8] [9] [10], quantum entanglement and quantum information [11]-[18]. At present, we are still lacking a deep understanding of photon and although some progress has been achieved, Einstein's light quanta question has not received a complete answer. In history, the research of photon nature obtained lots of achievements and promoted the creation of relativity and quantum theory. In 2003, Zajonc said our ignorance of photon quanta was then about Einstein [19].

In this paper, we have proposed a new single photon theory, which is the vector potential $\boldsymbol{A}$ rotation at the vertical motion direction of photon, it can produce the microscopic electric field and magnetic field, and they satisfy the Maxwell equations. We have calculated photon spin, momentum, energy, and found there are left-handed and right-handed photon, and they have wave-particle duality in the theory. We have given the relation between photon electric field intensity and energy. In addition, we study the multi-photon theory and the dynamics theory between photons interaction. On this basis, we have further given the compound structures of electron, proton, neutron, neutrino, $\pi$ meson, dark matter with the photon theory, and calculated their mass, charge and spin.

\section{The Single-Photon Structural Model}

We know that the photon carries energy-momentum and has spin. photon spin has always the same value for all photons (one unit of $\hbar$ ), whereas the energy and momentum can vary continuously from zero to infinity, and they are in relation to photon frequency, what is the photon frequency? In order to unify the photon energy, momentum, spin and frequency, we structure the photon structural model as: photon has the vector potential $\boldsymbol{A}$, and the vector potential $\boldsymbol{A}$ rotates at the vertical direction of photon motion, the rotation angle frequency $\omega$ is the photon angle frequency. It is shown in Figure 1.

There are four kinds of motion form as follows:

1) The photon moves along the $+Z$ direction, and the vector potential $A$ rotates clockwise, the rotation angular frequency is $\omega$, it is shown in Figure 2.

The vector potential $\boldsymbol{A}$ is 


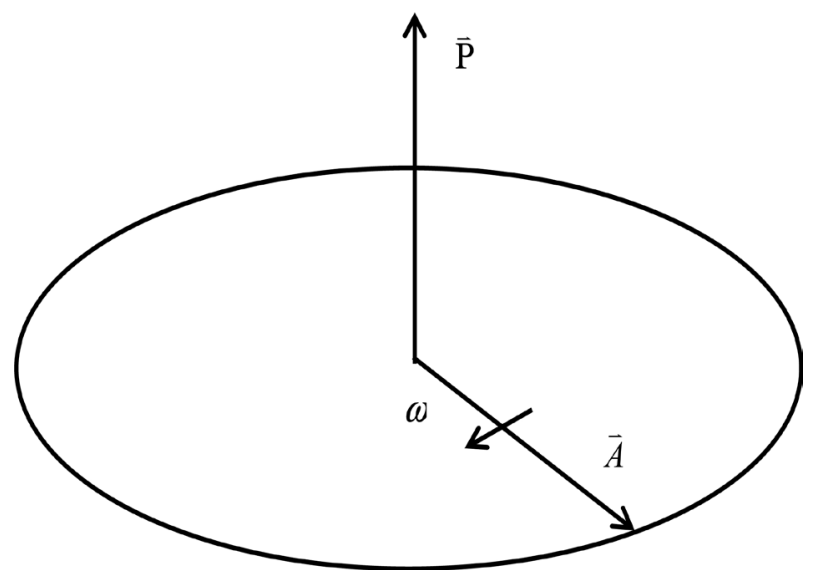

Figure 1. The photon structural figure, the vector potential $\boldsymbol{A}$ rotates at the vertical direction of photon motion.

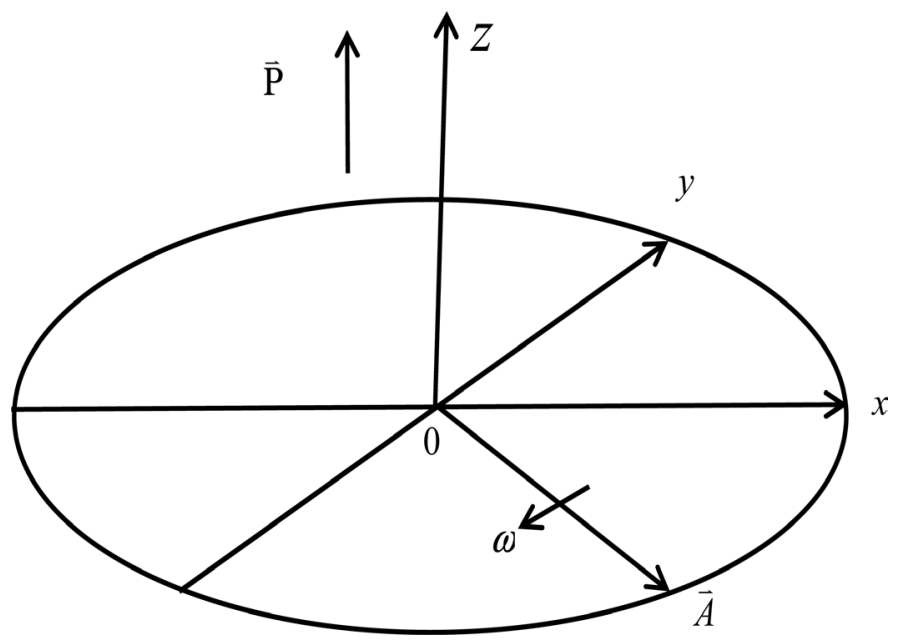

Figure 2. The photon moves along the $+z$ direction, and the vector potential $\boldsymbol{A}$ rotates clockwise, the rotation angular frequency is $\omega$.

$$
\boldsymbol{A}=A_{0}\left(\cos (\omega t-k z) \boldsymbol{e}_{x}-\sin (\omega t-k z) \boldsymbol{e}_{y}\right),
$$

with $A_{0}=|\boldsymbol{A}|$, and $k=\omega / c$. The photon vector potential $\boldsymbol{A}$ rotation should generate photon electric field and magnetic field, they are

$$
\begin{gathered}
\boldsymbol{E}=-\frac{\partial \boldsymbol{A}}{\partial t}=A_{0} \omega\left(\sin (\omega t-k z) \boldsymbol{e}_{x}+\cos (\omega t-k z) \boldsymbol{e}_{y}\right), \\
\boldsymbol{B}=\nabla \times \boldsymbol{A}=-k A_{0}\left(\cos (\omega t-k z) \overrightarrow{\boldsymbol{e}}_{x}-\sin (\omega t-k z) \boldsymbol{e}_{y}\right)=-k \boldsymbol{A},
\end{gathered}
$$

where $\boldsymbol{e}_{x}, \boldsymbol{e}_{y}$ and $\boldsymbol{e}_{z}$ are the unit vector of $x$ axis, $y$ axis and $z$ axis.

With Equation (1), we obtain the equations of vector potential $A$

$$
\nabla^{2} \boldsymbol{A}-\mu_{0} \varepsilon_{0} \frac{\partial^{2} \boldsymbol{A}}{\partial t^{2}}=0, \quad \nabla \cdot \boldsymbol{A}=0 .
$$

From Equations (1)-(4), we find the photon electric field $\boldsymbol{E}$ and magnetic field $\vec{B}$ satisfy Maxwell equations

$$
\nabla \cdot \boldsymbol{E}=0
$$




$$
\begin{gathered}
\nabla \cdot \boldsymbol{B}=0, \\
\nabla \times \boldsymbol{E}=-\frac{\partial \boldsymbol{B}}{\partial t}, \\
\nabla \times \boldsymbol{B}=\mu_{0} \varepsilon_{0} \frac{\partial \boldsymbol{E}}{\partial t} .
\end{gathered}
$$

With Equations ((2) and (3)), we find $|\boldsymbol{E}|=c|\boldsymbol{B}|$ and $\boldsymbol{B}=\frac{1}{c} \frac{\boldsymbol{k}}{\boldsymbol{k}} \times \boldsymbol{E}$. For the photon, its vector potential $\boldsymbol{A}$, electric field $\boldsymbol{E}$ and magnetic field $\boldsymbol{B}$ are distributed in minimal local of photon size, which are different from the macroscopic electromagnetic field, they can distribute in limited and infinite area. We call photon electric field (2) and magnetic field (3) as microscopic electric field and magnetic field, the Equations (6)-(8) as microscopic Maxwell equations. The Equations (1)-(8) describe the photon as a wave.

With the photon vector potential $\boldsymbol{A}$, electric field $\boldsymbol{E}$ and magnetic field $\boldsymbol{B}$, we can calculate photon energy, momentum and spin.

The photon energy $w_{\omega}$ is

$$
w_{\omega}=\varepsilon_{0} \int_{V_{\omega}} \boldsymbol{E}^{2} \mathrm{~d} \tau=\varepsilon_{0} A_{0}^{2} \omega^{2} V_{\omega}=\hbar \omega
$$

From Equation (9), we can obtain the microscopic expression of Planck constant $\hbar$

$$
\hbar=\varepsilon_{0} A_{0}^{2} \omega V_{\omega},
$$

where $V_{\omega}$ is photon volume.

the photon momentum $\boldsymbol{p}$ is

$$
\boldsymbol{p}=\varepsilon_{0} \int_{V_{\omega}} \boldsymbol{E} \times \boldsymbol{B} \mathrm{d} \tau=\varepsilon_{0} A_{0}^{2} \frac{\omega^{2}}{C} V_{\omega} \boldsymbol{e}_{z}=\frac{\hbar \omega}{c} \boldsymbol{e}_{z}=\hbar k \boldsymbol{e}_{z},
$$

the photon spin $S$ is

$$
\boldsymbol{S}=\varepsilon_{0} \int_{V_{\omega}} \boldsymbol{E} \times \boldsymbol{A} \mathrm{d} \tau=-\varepsilon_{0} A_{0}^{2} \omega V_{\omega} \boldsymbol{e}_{z}=-\hbar \boldsymbol{e}_{z},
$$

with Equations ((2) and (9)), we can give the relation between photon electric field intensity and energy, it is

$$
E=\sqrt{\frac{\hbar \omega}{\varepsilon_{0} V_{\omega}}}
$$

By the rotation of photon vector potential, we can obtain all information of photon, such as photon electric field, magnetic field, energy, momentum and spin, and the photon frequency (the vector potential rotation frequency). The Equation (10) gives out the microscopic expression of the Planck constant $\hbar$, it is in relation to vector potential amplitude $A_{0}$, vector potential rotation frequency $\omega$ and photon volume $V_{\omega}$. From Equation (12), we find the photon spin $S=1$, and when photon vector potential $\boldsymbol{A}$ rotates in the clockwise direction, photon spin $\boldsymbol{S}$ and its momentum $\boldsymbol{p}$ are in the opposite direction, it is called right-handed photon. The vector potential $\boldsymbol{A}$, electric field $\boldsymbol{E}$, magnetic field $\boldsymbol{B}$, spin $\boldsymbol{S}$ and momentum $\boldsymbol{p}$ are shown in Figure 3. Their 


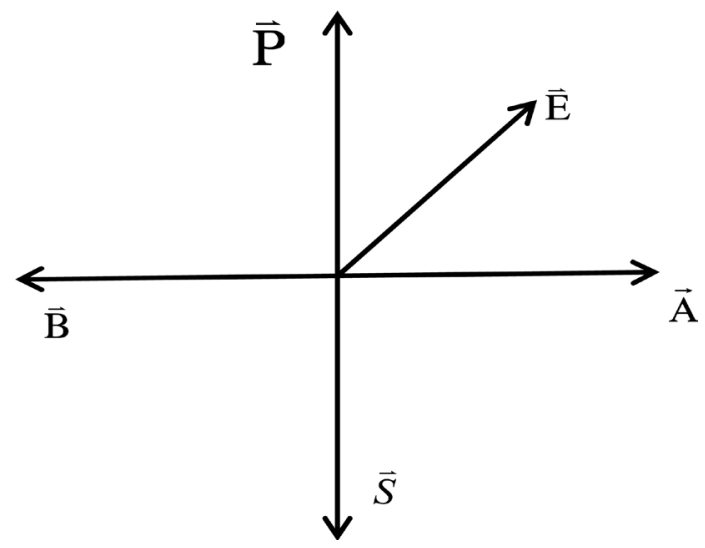

Figure 3. The relation of photon vector potential $\boldsymbol{A}$, electric field $\boldsymbol{E}$, magnetic field $\boldsymbol{B}$, spin $s$ and momentum $p$.

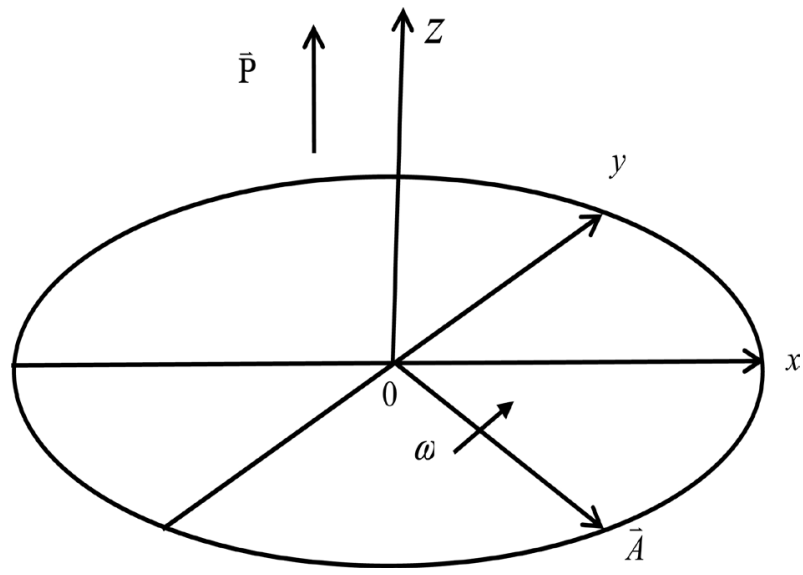

Figure 4. The photon moves along the $+Z$ direction, and the vector potential $A$ rotates anticlockwise, the rotation angular frequency is $\omega$.

relationship are: $\boldsymbol{E} \perp \boldsymbol{A}$ and $\boldsymbol{E}$ lag $\boldsymbol{A}$ angle $\frac{\pi}{2}, \boldsymbol{B} \|-\boldsymbol{A}, \boldsymbol{B} \perp \boldsymbol{E}$, they are in the same plane, and are clockwise rotation at angular frequency $\omega$, the $\boldsymbol{S}$ and $\boldsymbol{p}$ are perpendicular to the plane. The Equations (9)-(12) describe the photon as a particle. So, photon has wave-particle duality in the theory.

2) The photon moves along the $+z$ direction, and the vector potential $\boldsymbol{A}$ rotates anticlockwise, the rotation angular frequency is $\omega$, it is shown in Figure 4.

The photon vector potential $\boldsymbol{A}$ is

$$
\boldsymbol{A}=A_{0}\left(\cos (\omega t-k z) \boldsymbol{e}_{x}+\sin (\omega t-k z) \boldsymbol{e}_{y}\right),
$$

the vector potential $\boldsymbol{A}$ rotation should generate photon electric field and magnetic field, they are

$$
\begin{gathered}
\boldsymbol{E}=-\frac{\partial \boldsymbol{A}}{\partial t}=A_{0} \omega\left(\sin (\omega t-k z) \boldsymbol{e}_{x}-\cos (\omega t-k z) \boldsymbol{e}_{y}\right), \\
\boldsymbol{B}=\nabla \times \boldsymbol{A}=k A_{0}\left(\cos (\omega t-k z) \boldsymbol{e}_{x}+\sin (\omega t-k z) \boldsymbol{e}_{y}\right)=k \boldsymbol{A},
\end{gathered}
$$

and photon energy, momentum and spin are 


$$
\begin{gathered}
w_{\omega}=\varepsilon_{0} \int_{V_{\omega}} \boldsymbol{E}^{2} \mathrm{~d} \tau=\varepsilon_{0} A_{0}^{2} \omega^{2} V_{\omega}=\hbar \omega, \\
\boldsymbol{p}=\varepsilon_{0} \int_{V_{\omega}} \boldsymbol{E} \times \boldsymbol{B} \mathrm{d} \tau=\varepsilon_{0} A_{0}^{2} \frac{\omega^{2}}{c} V_{\omega} \boldsymbol{e}_{z}=\frac{\hbar \omega}{c} \boldsymbol{e}_{z}=\hbar k \boldsymbol{e}_{z}, \\
\boldsymbol{S}=\varepsilon_{0} \int_{V_{\omega}} \boldsymbol{E} \times \boldsymbol{A} \mathrm{d} \tau=\varepsilon_{0} A_{0}^{2} \omega V_{\omega} \boldsymbol{e}_{z}=\hbar \boldsymbol{e}_{z} .
\end{gathered}
$$

The vector potential $\boldsymbol{A}$, electric field $\boldsymbol{E}$, magnetic field $\boldsymbol{B}$, momentum $\boldsymbol{p}$ and spin $\boldsymbol{S}$ are shown in Figure 5. Their relationship are: $\boldsymbol{E} \perp \boldsymbol{A}$ and $\boldsymbol{E}$ ahead $\boldsymbol{E}$ angle $\frac{\pi}{2}, \boldsymbol{B} \| \boldsymbol{A}, \boldsymbol{B} \perp \boldsymbol{E}$, they are in the same plane, and are anticlockwise rotation at angular frequency $\omega$. The spin $S$ and momentum $\boldsymbol{p}$ are in the same direction, it is called left-handed photon.

3) The photon moves along the $-z$ direction, and the vector potential $A$ rotates clockwise, the rotation angular frequency is $\omega$. It has electric field, magnetic field, energy, momentum and spin, and the spin $S$ and momentum $\boldsymbol{p}$ are in the same direction, it is left-handed photon.

4) The photon moves along the $-z$ direction, and the vector potential $A$ rotates anticlockwise, the rotation angular frequency is $\omega$, it has electric field, magnetic field, energy, momentum and spin, and the spin $S$ and momentum $\boldsymbol{p}$ are in the opposite direction, it is right-handed photon.

For the four kinds of motion form of photon, we can find the vector potential $\boldsymbol{A}$, electric field $\boldsymbol{E}$ and magnetic field $\boldsymbol{B}$ are in the same plane and orthogonality, the spin $S$ and momentum $\boldsymbol{p}$ are perpendicular to the plane. The $\boldsymbol{A}, \boldsymbol{E}$ and $\boldsymbol{B}$ are all rotation at angular frequency $\omega$, but the relative direction between them are different, and the $S$ and $\boldsymbol{p}$ direction are also different at the four kinds of motion form of photon.

\section{The Photon Dynamics Theory}

We know that photon has electric field, magnetic field and spin, which lead to interaction between the photons. For the two photons, there are interaction between their electric fields, magnetic fields and spins, when the directions of electric field $\bar{E}$, magnetic field $\bar{B}$ and spin $\bar{S}$ of two photons are same

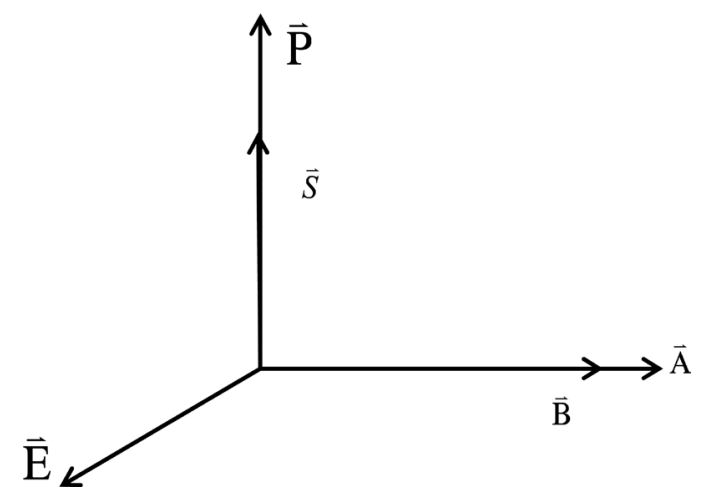

Figure 5. The relation of photon vector potential $\boldsymbol{A}$, electric field $\boldsymbol{E}$, magnetic field $\boldsymbol{B}$, spin $S$ and momentum $\boldsymbol{p}$. 
(opposite), there are attractive force (repulsive force) between the two photons, the interaction potential energy between the two photons can be written as:

$$
\begin{aligned}
& V_{E}=g_{E} \bar{E} \cdot \bar{E}, \\
& V_{B}=g_{B} \bar{B} \cdot \bar{B},
\end{aligned}
$$

and

$$
V_{S}=g_{S} \bar{S} \cdot \bar{S}
$$

the total interaction potential energy is

$$
\begin{aligned}
V & =V_{E}+V_{B}+V_{S}=g_{E} \bar{E} \cdot \bar{E}+g_{B} \bar{B} \cdot \bar{B}+g_{S} \bar{S} \cdot \bar{S} \\
& =\left(g_{E}+\frac{g_{B}}{c^{2}}\right) \bar{E} \cdot \bar{E}+g_{S} \bar{S} \cdot \bar{S} \\
& =g_{E B} \bar{E} \cdot \bar{E}+g_{S} \bar{S} \cdot \bar{S} .
\end{aligned}
$$

where $V_{E}, V_{B}$ and $V_{S}$ are the potential energy of electric field, magnetic field and spin between two photons, $g_{E}, g_{B}$ and $g_{S}$ are the interaction constants of electric field, magnetic field and spin.

With the photon dynamics theory, we can give the following results:

1) Two photons can condensed into mass particle

For the two photons of vector potential $\boldsymbol{A}$ and $-\boldsymbol{A}$, their rotation angle frequency $\omega$, and motor direction are same, they can condense into photoncouple, it is shown in Figure 6. With Equation (23), we can find the two photons should be in the balance state, since they suffer the electromagnetic attraction and the spin repulsion. The photon-couple total vector potential, total electric field magnetic field, total spin and momentum all are zero, and the two photons energy should be absolutely transformed into mass of photon-couple, it is

$$
m_{\omega}=\frac{2 \hbar \omega}{c^{2}} .
$$

where $c$ is the light velocity, the condensed photon-couple is graviton, which should form a particle mass and gravitational field.

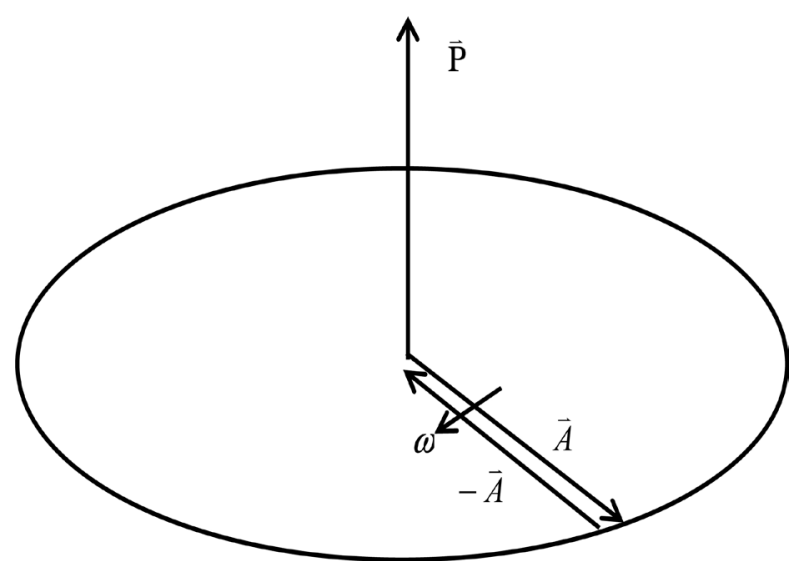

Figure 6. The two-photon of vector potentials $A$ and $-\boldsymbol{A}$ rotate at angle frequency $\omega$, they condensed into photon couple, and the photon couple mass is $m_{\omega}=\frac{2 \hbar \omega}{c^{2}}$. 
2) Two photons can generate a new photon

For the two photons of vector potential $\boldsymbol{A}$, their rotation angle frequency $\omega$ and motor direction are same. With Equation (23), we can find the two photons should not be in the balance state, since they suffer the electromagnetic and the spin repulsion, but they can generate a new photon of vector potential $A^{\prime}$ and rotation angle frequency $2 \omega$, i.e., $\gamma(\boldsymbol{A}, \omega)+\gamma(\boldsymbol{A}, \omega) \rightarrow \gamma\left(\boldsymbol{A}^{\prime}, 2 \omega\right)$.

\section{The Multi-Photon Theory}

In the above, we have given the single-photon structural model. In the following, we shall study the multi-photon process with the photon dynamics theory.

1) $\gamma(\boldsymbol{A}, \omega)+\gamma(\boldsymbol{A}, \omega) \rightarrow \gamma\left(\boldsymbol{A}^{\prime}, 2 \omega\right)$

The process $\gamma(\boldsymbol{A}, \omega)+\gamma(\boldsymbol{A}, \omega) \rightarrow \gamma\left(\boldsymbol{A}^{\prime}, 2 \omega\right)$ denotes the two photons that their motor direction, vector-potential $\boldsymbol{A}$ magnitude, rotation direction and rotation angular frequency $\omega$ are all the same, they can form a new photon that angular frequency and volume become double. In the process, the momentum and energy are conservative. With Equation (9), we have

$$
\varepsilon_{0}\left(A_{0} \omega\right)^{2} V_{\omega}+\varepsilon_{0}\left(A_{0} \omega\right)^{2} V_{\omega}=\varepsilon_{0}\left(A_{0}^{\prime} 2 \omega\right)^{2} 2 V_{\omega},
$$

by Equation (25), we obtain

$$
A_{0}^{\prime} 2 \omega=A_{0} \omega=e_{0}
$$

with the Planck constant $\hbar=\varepsilon_{0} A_{0}^{2} \omega V_{\omega}=\varepsilon_{0}\left(A_{0} \omega\right)^{2} \frac{V_{\omega}}{\omega}$, we get

$$
\frac{V_{\omega}}{\omega}=g_{0},
$$

and

$$
A_{0} V_{\omega}=e_{0} g_{0} .
$$

where $e_{0}$ and $g_{0}$ are the constants, and the Planck constant can be written as $\hbar=\varepsilon_{0}\left(e_{0}\right)^{2} g_{0}$.

2) $\gamma\left(\boldsymbol{A}_{1}, \omega_{1}\right)+\gamma\left(\boldsymbol{A}_{2}, \omega_{2}\right) \rightarrow \gamma\left(\boldsymbol{A}_{3},\left(\omega_{1}+\omega_{2}\right)\right)$

The process $\gamma\left(\boldsymbol{A}_{1}, \omega_{1}\right)+\gamma\left(\boldsymbol{A}_{2}, \omega_{2}\right) \rightarrow \gamma\left(\boldsymbol{A}_{3},\left(\omega_{1}+\omega_{2}\right)\right)$ denotes the two photons that vector-potential are $A_{1}$ and $A_{2}$, rotation angular frequency are $\omega_{1}$ and $\omega_{2}$ and their motor direction, vector-potential rotation direction are same, they can form a new photon.

With the energy conservation and Equation (9), we have

$$
\varepsilon_{0}\left(A_{01} \omega_{1}\right)^{2} V_{\omega_{1}}+\varepsilon_{0}\left(A_{02} \omega_{2}\right)^{2} V_{\omega_{2}}=\varepsilon_{0}\left(A_{03}\left(\omega_{1}+\omega_{2}\right)\right)^{2} V_{\omega_{1}+\omega_{2}},
$$

i.e.,

$$
A_{01} \omega_{1}=A_{02} \omega_{2}=A_{03}\left(\omega_{1}+\omega_{2}\right),
$$

and

$$
V_{\omega_{1}+\omega_{2}}=V_{\omega_{1}}+V_{\omega_{2}} .
$$

3) $\gamma\left(\boldsymbol{A}_{1}, \omega_{1}\right)+\gamma\left(\boldsymbol{A}_{2}, \omega_{2}\right)+\cdots+\gamma\left(\boldsymbol{A}_{n}, \omega_{n}\right) \rightarrow \gamma\left(\boldsymbol{A}_{n+1},\left(\omega_{1}+\omega_{2}+\cdots+\omega_{n}\right)\right)$ 
The process

$\gamma\left(\boldsymbol{A}_{1}, \omega_{1}\right)+\gamma\left(\boldsymbol{A}_{2}, \omega_{2}\right)+\cdots+\gamma\left(\boldsymbol{A}_{n}, \omega_{n}\right) \rightarrow \gamma\left(\boldsymbol{A}_{n+1},\left(\omega_{1}+\omega_{2}+\cdots+\omega_{n}\right)\right)$ denotes the $n$ photons that vector-potential are $A_{i}(i=1,2, \cdots, n)$, rotation angular frequency are $\omega_{i}(i=1,2, \cdots, n)$ and their motor direction, vector-potential rotation direction are same, they can form a new photon.

With the energy conservation and Equation (9), we have

$$
\begin{aligned}
& \varepsilon_{0}\left(\left(A_{01} \omega_{1}\right)^{2} V_{\omega_{1}}+\left(A_{02} \omega_{2}\right)^{2} V_{\omega_{2}}+\cdots+\left(A_{0 n} \omega_{n}\right)^{2} V_{\omega_{n}}\right) \\
& =\varepsilon_{0}\left(A_{0 n+1}\left(\omega_{1}+\omega_{2}+\cdots+\omega_{n}\right)\right)^{2} V_{\omega_{1}+\omega_{2}+\cdots+\omega_{n}},
\end{aligned}
$$

i.e.,

$$
A_{01} \omega_{1}=A_{02} \omega_{2}=\cdots=A_{0 n+1}\left(\omega_{1}+\omega_{1}+\cdots+\omega_{n}\right),
$$

and

$$
V_{\omega_{1}+\omega_{2}+\cdots+\omega_{n}}=V_{\omega_{1}}+V_{\omega_{2}}+\cdots+V_{\omega_{n}} .
$$

4) $\gamma(\boldsymbol{A}, \omega)+\gamma(-\boldsymbol{A}, \omega) \rightarrow d$

The process $\gamma(\boldsymbol{A}, \omega)+\gamma(-\boldsymbol{A}, \omega) \rightarrow d$ denotes the two photons that their motor direction, vector-potential $\boldsymbol{A}$ magnitude, rotation direction and rotation angular frequency $\omega$ all are same, but their vector-potential $\boldsymbol{A}$ direction is opposite, they can form a neutral particle $d$, its mass $m_{d}=\frac{2 \hbar \omega}{c^{2}}$, charge $q=0$ and spin $s=0$, the neutral particle $d$ is a dark particle.

\section{The Macroscopic Electromagnetic Field Structural Model}

In the photon structural model, we have obtained the electric field $\boldsymbol{E}_{R}$ and $\boldsymbol{E}_{L}$ for the right-handed and left-handed photon, they are

$$
\boldsymbol{E}_{R}=A_{0} \omega\left(\sin (\omega t-k z) \boldsymbol{e}_{x}+\cos (\omega t-k z) \boldsymbol{e}_{y}\right),
$$

and

$$
\boldsymbol{E}_{L}=A_{0} \omega\left(\sin (\omega t-k z) \boldsymbol{e}_{x}-\cos (\omega t-k z) \boldsymbol{e}_{y}\right),
$$

they can be written as the plural form

$$
\boldsymbol{E}=A_{0} \omega \mathrm{e}^{i(\omega t-k z+\varphi)},
$$

its real part and imaginary part express the electric field of $\boldsymbol{e}_{y}$ and $\boldsymbol{e}_{x}$ components, respectively. The electric field of linear polarization photon is the superposition of right-handed and left-handed photon electric field $\boldsymbol{E}_{R}$ and $\boldsymbol{E}_{L}$, it is

$$
\boldsymbol{E}_{/ /}=\boldsymbol{E}_{R}+\boldsymbol{E}_{L}=2 A_{0} \omega \sin (\omega t-k z) \boldsymbol{e}_{x},
$$

or

$$
\boldsymbol{E}_{/ /}=\boldsymbol{E}_{R}-\boldsymbol{E}_{L}=2 A_{0} \omega \cos (\omega t-k z) \boldsymbol{e}_{y} .
$$

The macroscopic electromagnetic field are the the superposition of all photons electromagnetic field, they are 


$$
\boldsymbol{E}(\boldsymbol{r}, t)=\sum_{i} \boldsymbol{E}_{i}(\boldsymbol{r}, t), \quad \boldsymbol{B}(\boldsymbol{r}, t)=\sum_{i} \boldsymbol{B}_{i}(\boldsymbol{r}, t),
$$

where $\boldsymbol{E}_{i}(\boldsymbol{r}, t)$ and $\boldsymbol{B}_{i}(\boldsymbol{r}, t)$ are the i-th photon electric field and magnetic field, and the continuous superposition form is

$$
\boldsymbol{E}(\boldsymbol{r}, t)=\int_{-\infty}^{+\infty} \boldsymbol{E}(\omega) \mathrm{e}^{i(\omega t-\boldsymbol{k} \cdot \boldsymbol{r})} \mathrm{d} \omega .
$$

The macroscopic electromagnetic field satisfy the Maxwell equations.

\section{The Electron Structural Theory}

Firstly, we define a new function $g(x)$, it satisfies

$$
g(x)= \begin{cases}0, & x \neq 0 \\ 1, & x=0\end{cases}
$$

obviously, $g(x)$ function form is

$$
g(x)=\frac{1}{2 \pi} \int_{0}^{2 \pi} \mathrm{e}^{i k x} \mathrm{~d} k .
$$

1) the electron structural

We think electron is made up of plenty of photons, which rotate around the ball center at different angular frequency $\omega$, and are sphere-symmetry distribution in space. These photons in electron are divided into two categories. One kind is the non-condensation photons, which their vector potentials $\boldsymbol{A}$ direction are same at the same space point $\boldsymbol{r}$, it is shown in Figure 7. The non-condensation photons form the electron electric field, charge and spin. Another kind is the condensation photons, which are formed by the two photons of vector potential $\boldsymbol{A}$ and $-\boldsymbol{A}$ condensed into photon-couple, and the condensation photon-couple form the electron mass.

For the photon of rotation angle frequency $\omega$, at $\theta$ angle, its vector potential $\boldsymbol{A}$ can be written as:

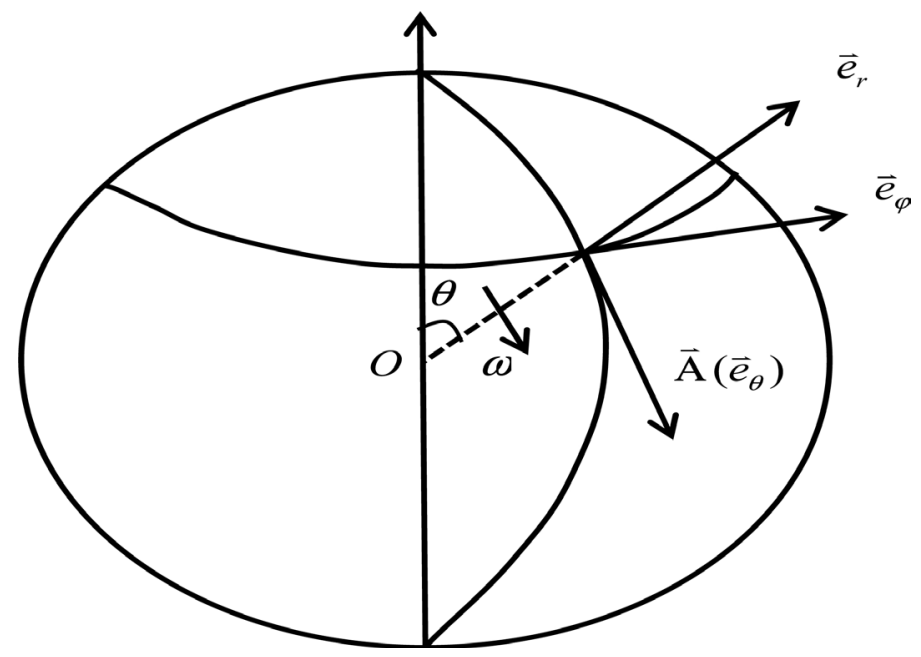

Figure 7. The electron structure figure, it is made up of plenty of photons, and the photons vector potentials $\boldsymbol{A}$ rotate around the ball center at different angular frequency $\omega$. 


$$
\boldsymbol{A}(t)=g(\theta-\omega t) \boldsymbol{A}_{0}=\frac{1}{2 \pi} A_{0} \int_{0}^{2 \pi} \mathrm{e}^{i k(\theta-\omega t)} \mathrm{d} k \boldsymbol{e}_{\theta} .
$$

At $\theta=\omega t$, the photon electric field and magnetic field are

$$
\begin{gathered}
\boldsymbol{E}_{\omega}=-\frac{\partial \boldsymbol{A}}{\partial t}=\frac{i}{2 \pi} A_{0} \omega \int_{0}^{2 \pi} k \mathrm{e}^{i k(\theta-\omega t)} \mathrm{d} k \boldsymbol{e}_{\theta}=i \omega \pi A_{0} \boldsymbol{e}_{\theta}=-\omega \pi A_{0} \boldsymbol{e}_{r}, \\
\boldsymbol{B}_{\omega}=\frac{1}{c}\left|\boldsymbol{E}_{\omega}\right| \boldsymbol{e}_{\varphi},
\end{gathered}
$$

where $\boldsymbol{e}_{\theta}, \boldsymbol{e}_{r}$ and $\boldsymbol{e}_{\varphi}$ are the unit vectors in the $\theta, r$ and $\varphi$ direction, respectively. From Equations ((45) and (46)), we can find the photon in electron generate electric field along the $-\boldsymbol{e}_{r}$ direction, it is a emanative field and can be observed and tested, its magnetic field along the $\boldsymbol{e}_{\varphi}$ direction, it is a closed field, since the magnetic fields are symmetry distribution when the electron relative rest, it can not be observed and tested. when the electron is in movement, the magnetic fields sphere-symmetry distribution should be destroyed, it can be observed and tested, i.e., movement charge produces magnetic field.

2) the electron electric field and charge

With the electron structural and the photon electric field, we can calculate the electron electric field, and give the electron charge, the electron electric field can be written as:

$$
\boldsymbol{E}(r)=\int_{0}^{\infty} n(r, \omega) \boldsymbol{E}_{\omega} \mathrm{d} \omega
$$

where $n(r, \omega)$ is the non-condensable photon numbers distribution of unit frequency at space $r$ and frequency interval $\omega \rightarrow \omega+\mathrm{d} \omega$, we assume

$$
n(r, \omega)=\frac{a \omega \mathrm{e}^{-b \omega^{2}}}{2 \varepsilon_{0} \pi^{2} r^{2}},
$$

substituting Equations ((45) and (48)) into (47), we have

$$
\boldsymbol{E}(r)=-\frac{a e_{0}}{4 \pi \varepsilon_{0} b r^{2}} \boldsymbol{e}_{r},
$$

comparing with the Coulomb law $\boldsymbol{E}(r)=\frac{-Q}{4 \pi \varepsilon_{0} r^{2}} \boldsymbol{e}_{r}$, we obtain

$$
Q=\frac{a e_{0}}{b}
$$

For the electron charge $-Q$, its distribution function $n(r, \omega)$ is

$$
n(r, \omega)=\frac{b Q \omega \mathrm{e}^{-b \omega^{2}}}{2 \varepsilon_{0} \pi^{2} e_{0} r^{2}} .
$$

where $e_{0}=\omega A_{0}$ is a constant, and $b$ is a undetermined parameter. In electron, when the non-condensable photon distribution function is (51), we can obtain the electron charge $-Q$ and its Coulomb static electric field distribution.

3) the electron spin

In electron, the photon spin of rotation angle frequency $\omega$ is 


$$
\boldsymbol{S}_{\omega}=\varepsilon_{0} \int_{V_{\omega}} \boldsymbol{E}_{\omega} \times \boldsymbol{A}_{0} \mathrm{~d} \tau=-\varepsilon_{0} \pi \omega A_{0}^{2} V_{\omega} \boldsymbol{e}_{\varphi},
$$

we can define electron spin as the total spin of photon at the semicircle ( $\varphi=$ constant angle, $0 \leq r<\infty$ ), the definition is reasonable, since $\varphi$ equals different constant, the spin values are the same, it is

$$
\begin{aligned}
\boldsymbol{S} & =\int_{0}^{\infty} \int_{0}^{\infty} \boldsymbol{S}_{\omega} m(r, \omega) \mathrm{d}\left(\frac{\pi r^{2}}{2}\right) \mathrm{d} \omega \\
& =-\pi^{2} \boldsymbol{e}_{\varphi} \int_{0}^{\infty} \int_{0}^{\infty} \varepsilon_{0} \omega A_{0}^{2} V_{\omega} r m(r, \omega) \mathrm{d} r d \omega \\
& =-\pi^{2} \hbar \boldsymbol{e}_{\varphi} \int_{0}^{\infty} \int_{0}^{\infty} r m(r, \omega) \mathrm{d} r \mathrm{~d} \omega,
\end{aligned}
$$

where $m(r, \omega)$ is the non-condensable photon numbers distribution of unit area and unit frequency at $\omega \rightarrow \omega+\mathrm{d} \omega, r \rightarrow r+\mathrm{d} r$ half donut. when $\int_{0}^{\infty} r m(r, \omega) \mathrm{d} r \mathrm{~d} \omega=\frac{1}{2 \pi^{2}}$, the electronic spin $S=-\frac{\hbar}{2}$, we assume

$$
m(r, \omega)=a \mathrm{e}^{-h r^{2}} \omega \mathrm{e}^{-b \omega^{2}},
$$

the integral

$$
\int_{0}^{\infty} \int_{0}^{\infty} r m(r, \omega) \mathrm{d} r \mathrm{~d} \omega=\frac{a}{4 b h}=\frac{1}{2 \pi^{2}},
$$

i.e.,

$$
a=\frac{2 b h}{\pi^{2}},
$$

the photon numbers distribution function is

$$
m(r, \omega)=\frac{2 b h}{\pi^{2}} \omega \mathrm{e}^{-h r^{2}-b \omega^{2}} .
$$

where $h$ and $b$ are the undetermined parameters. In electron, when the photon distribution function is Equation (57), the electronic spin $S=-\frac{\hbar}{2}$ can be obtained.

In Figure 7, if electron turns along the $z$ axis, the photon vector potential $\boldsymbol{A}$ should become

$$
\boldsymbol{A}(t)=g(\theta+\omega t)\left(-\boldsymbol{A}_{0}\right)=-\frac{1}{2 \pi} A_{0} \int_{0}^{2 \pi} \mathrm{e}^{i k(\theta+\omega t)} \mathrm{d} k \boldsymbol{e}_{\theta},
$$

At $\theta=-\omega t$, the photon electric field and magnetic field are

$$
\begin{gathered}
\boldsymbol{E}_{\omega}=-\frac{\partial \boldsymbol{A}}{\partial t}=\frac{i}{2 \pi} A_{0} \omega \int_{0}^{2 \pi} k \mathrm{e}^{i k(\theta+\omega t)} \mathrm{d} k \boldsymbol{e}_{\theta}=i \omega \pi A_{0} \boldsymbol{e}_{\theta}=-\omega \pi A_{0} \boldsymbol{e}_{r}, \\
\boldsymbol{B}_{\omega}=\frac{1}{C}\left|\boldsymbol{E}_{\omega}\right| \boldsymbol{e}_{\varphi} .
\end{gathered}
$$

In electron, the photon spin of rotation angle frequency $\omega$ is

$$
\boldsymbol{S}_{\omega}=\varepsilon_{0} \int_{V_{\omega}} \boldsymbol{E}_{\omega} \times \boldsymbol{A}_{0} \mathrm{~d} \tau=\varepsilon_{0} \pi \omega A_{0}^{2} V_{\omega} \boldsymbol{e}_{\varphi} .
$$

With Equation (53), we obtain the electron spin $S=\frac{\hbar}{2}$. Therefore, when the 
electron turns along the symmetry axis, its spin should be changed from $S=-\frac{\hbar}{2}\left(S=\frac{\hbar}{2}\right)$ to $S=\frac{\hbar}{2} \quad\left(S=-\frac{\hbar}{2}\right)$, i.e., the electron spin projection change comes from the electron rolling-over along the symmetry axis. In electron, the photon vector potential $\boldsymbol{A}$ are (44) and (58), respectively, the electron spin projections are $\frac{\hbar}{2}$ and $-\frac{\hbar}{2}$.

4) the electronic mass

The two photons of vector potential $\boldsymbol{A}$ and $-\boldsymbol{A}$ can condense into photoncouple when they rotate at the same angle frequency $\omega$, it is shown in Figure 6 . The two photon energy should be transform mass, and the mass is $m_{\omega}=\frac{2 \hbar \omega}{c^{2}}$, where $c$ is the light velocity. The electronic mass is the sum of all condensation photon-couples mass, it can be written as:

$$
m_{e}=\iint m_{\omega} \rho_{e}(r, \omega) \mathrm{d} V \mathrm{~d} \omega,
$$

where $\rho_{e}(r, \omega)$ is the condensation photon-couple numbers density of unit volume and unit frequency at $r \rightarrow r+\mathrm{d} r$ and $\omega \rightarrow \omega+\mathrm{d} \omega, m_{\omega}$ is the condensation photon-couple mass, we assume

$$
\rho_{e}(r, \omega)=g n_{e} \mathrm{e}^{-a_{0} r^{3}} \omega \mathrm{e}^{-b_{0} \omega^{2}},
$$

the electronic mass is

$$
\begin{aligned}
m_{e} & =\iint m_{\omega} \rho_{e}(r, \omega) \mathrm{d} V \mathrm{~d} \omega \\
& =4 \pi \iint \frac{2 n_{e} \hbar \omega}{c^{2}} r^{2} g \mathrm{e}^{-a_{0} r^{3}} \omega \mathrm{e}^{-b_{0} \omega^{2}} \mathrm{~d} r \mathrm{~d} \omega \\
& =\frac{8 \pi n_{e} \hbar g}{c^{2}} \int_{0}^{\infty} r^{2} \mathrm{e}^{-a_{0} r^{3}} \mathrm{~d} r \int_{0}^{\infty} \omega^{2} \mathrm{e}^{-b_{0} \omega^{2}} \mathrm{~d} \omega \\
& =\frac{2 \pi n_{e} \hbar g}{3 a_{0} b_{0} \sqrt{b_{0}} c^{2}} .
\end{aligned}
$$

where $a_{0}, b_{0}, n_{e}$ and $g$ are undetermined parameters, the electronic mass is related to them.

\section{The Proton, Neutron and Neutrino Structural Theory}

With the electron structural theory, we can give the proton, neutron and neutrino structural, they are also made up of lots of photons, and they have a definite spatial distribution.

1) the proton structural theory

Comparing proton with electron, they have the same spin, but their charges are $+Q$ and $-Q$, and their masses are $m_{p}>m_{e}$. The positive and negative of charges are related to the electric field direction, the electron charge is $-Q$, its electric field is $-e_{r}$ direction. The proton charge is $+Q$, its electric field is $e_{r}$ direction. In proton, its photon vector potential rotation direction should be opposite to electron's, it is

$$
\boldsymbol{A}(t)=g(\theta+\omega t) \boldsymbol{A}_{0}=\frac{1}{2 \pi} A_{0} \int_{0}^{2 \pi} \mathrm{e}^{i k(\theta+\omega t)} \mathrm{d} k \boldsymbol{e}_{\theta},
$$


when proton turns along the $z$ axis, its photon vector potential $\boldsymbol{A}$ should become

$$
\boldsymbol{A}(t)=g(\theta-\omega t)\left(-\boldsymbol{A}_{0}\right)=-\frac{1}{2 \pi} A_{0} \int_{0}^{2 \pi} \mathrm{e}^{i k(\theta-\omega t)} \mathrm{d} k \boldsymbol{e}_{\theta} .
$$

For the photon vector potentials (65) and (66), their photon electric field all are

$$
\boldsymbol{E}_{\omega}=-\frac{\partial \boldsymbol{A}}{\partial t}=-i \omega \pi A_{0} \boldsymbol{e}_{\theta}=\omega \pi A_{0} \boldsymbol{e}_{r} .
$$

With Equations (47), (48) and (67), we can obtain the proton electric field $\boldsymbol{E}(r)=\frac{Q}{4 \pi \varepsilon_{0} r^{2}} \boldsymbol{e}_{r}$ and proton charge $Q=\frac{a e_{0}}{b}$. Similar to the electron spin calculation, the two vector potentials (65) and (66) are corresponding to the proton spin projection $\frac{\hbar}{2}$ and $-\frac{\hbar}{2}$.

It is similar to calculation electron mass, the proton mass is from the condensation photon-couple numbers density $\rho_{p}(r, \omega)$, it is

$$
\rho_{p}(r, \omega)=g n_{p} \mathrm{e}^{-a_{0} r^{3}} \omega \mathrm{e}^{-b_{0} \omega^{2}},
$$

then the proton mass is

$$
m_{p}=\iint m_{\omega} \rho_{p}(r, \omega) \mathrm{d} V \mathrm{~d} \omega=\frac{2 \pi n_{p} \hbar g}{3 a_{0} b_{0} \sqrt{b_{0}} c^{2}} .
$$

2) the neutron structural theory

For the neutron, it is made up of vast photons. It has the same spin with electron and proton, but it has not charge, and its mass is $m_{n} \approx m_{p}$. We know particle mass is from the distribution of condensation photon-couple, particle spin and charge are from the distribution of non-condensable photons. The particle diffuse electric field can be observed, it has the charge effect, the particle annular electric field can not be observed, it has not the charge effect. In neutron, its photon vector potential $\boldsymbol{A}$ should be

$$
\boldsymbol{A}(t)=g(\theta-\omega t) \boldsymbol{A}_{0}=\frac{1}{2 \pi} A_{0} \int_{0}^{2 \pi} \mathrm{e}^{i k(\theta-\omega t)} \mathrm{d} k \boldsymbol{e}_{r},
$$

when neutron turns along the $z$ axis, its photon vector potential $\boldsymbol{A}$ should become

$$
\boldsymbol{A}(t)=g(\theta+\omega t) \boldsymbol{A}_{0}=\frac{1}{2 \pi} A_{0} \int_{0}^{2 \pi} \mathrm{e}^{i k(\theta+\omega t)} \mathrm{d} k \boldsymbol{e}_{r} .
$$

For the photon vector potentials (70) and (71), their photon electric field are

$$
\boldsymbol{E}_{\omega}=-\frac{\partial \boldsymbol{A}}{\partial t}=i \omega \pi A_{0} \boldsymbol{e}_{r}=\omega \pi A_{0} \boldsymbol{e}_{\theta} .
$$

and

$$
\boldsymbol{E}_{\omega}=-\frac{\partial \boldsymbol{A}}{\partial t}=-i \omega \pi A_{0} \boldsymbol{e}_{r}=-\omega \pi A_{0} \boldsymbol{e}_{\theta},
$$


the photon electric fields along $\boldsymbol{e}_{\theta}$ or $-\boldsymbol{e}_{\theta}$ direction, the neutron electric field is the annular distribution, which can not be observed, it has not the charge effect. Hence, neutron has not charge. As $\boldsymbol{B}_{\omega}=\frac{1}{c} \frac{\boldsymbol{k}}{k} \times \boldsymbol{E}_{\omega}=0$, neutron has not magnetic field distribution. Similar to the electron spin calculation, the two vector potentials (70) and (71) are corresponding to neutron two spin projection $\frac{\hbar}{2}$ and $-\frac{\hbar}{2}$. In neutron, the condensation photon-couple numbers density $\rho_{n}(r, \omega)$ is

$$
\rho_{n}(r, \omega)=g n_{n} \mathrm{e}^{-a_{0} r^{3}} \omega \mathrm{e}^{-b_{0} \omega^{2}},
$$

then the neutron mass is

$$
m_{n}=\iint m_{\omega} \rho_{n}(r, \omega) \mathrm{d} V \mathrm{~d} \omega=\frac{2 \pi n_{n} \hbar g}{3 a_{0} b_{0} \sqrt{b_{0}} c^{2}},
$$

the mass ratio of electron, proton and neutron is

$$
m_{e}: m_{p}: m_{n}=n_{e}: n_{p}: n_{n} .
$$

From Equation (76), we find the elementary particle mass is quantized.

3) the neutrino structural theory

Comparing neutrino with neutron, they have not charges, and spins are $\frac{1}{2}$, the neutron has mass, but the neutron has not mass. So, the photon couple numbers density of neutron is zero, and the non-condensable photons numbers density $n(r, \omega)$ (Equation (54)) is same with electron, proton and neutron, then they have the same spin. The neutrino photon vector potential $\boldsymbol{A}$ is (70) or (71), which is corresponding to neutrino spin projection $\frac{\hbar}{2}$ and $-\frac{\hbar}{2}$. If neutrino has less mass, its condensation photon-couple numbers density is not zero, it is should be detected further by experiment.

\section{The Meson $\pi^{0}, \pi^{+}$and $\pi^{-}$Structural Theory}

For the meson $\pi^{0}, \pi^{+}$and $\pi^{-}$, their spin $S=0$, mass $m_{\pi} \neq 0$, and charges $Q$ are $0, Q$ and $-Q$, respectively. By the research of electron, proton and neutron, we can give the $\pi$ meson photon structure.

1) The $\pi^{0}$ meson structure

Since the $\pi^{0}$ meson has not spin and charge, it has not the distribution of non-condensable photons, its mass $m_{\pi^{0}} \neq 0$, the condensation photon-couple numbers density $\rho_{\pi^{0}}(r, \omega)$ can be written as:

$$
\rho(r, \omega)=g n_{\pi^{0}} \mathrm{e}^{-a_{0} r^{3}} \omega \mathrm{e}^{-b_{0} \omega^{2}},
$$

and its mass is

$$
m_{\pi^{0}}=\iint m_{\omega} \rho_{\pi^{0}}(r, \omega) \mathrm{d} V \mathrm{~d} \omega=\frac{2 \pi n_{\pi^{0}} \hbar g}{3 a_{0} b_{0} \sqrt{b_{0}} c^{2}},
$$


2) The $\pi^{+}$meson structure

The $\pi^{+}$meson has mass and positive charge, but has not spin. We know the non-condensation photons distribution produce particle charge and spin, and the condensation photons distribution produce particle mass. So, the $\pi^{+}$meson should has two kinds of photon vector potentials, they are

$$
\boldsymbol{A}(t)=g(\theta+\omega t) \boldsymbol{A}_{0}=\frac{1}{2 \pi} A_{0} \int_{0}^{2 \pi} \mathrm{e}^{i k(\theta+\omega t)} \mathrm{d} k \boldsymbol{e}_{\theta}
$$

and

$$
\boldsymbol{A}(t)=\left((\theta-\omega t) \boldsymbol{A}_{0}=\frac{1}{2 \pi} A_{0} \int_{0}^{2 \pi} \mathrm{e}^{i k(\theta-\omega t)} \mathrm{d} k \boldsymbol{e}_{r}\right.
$$

The photon vector potential (79) produces $\pi^{+}$meson charge $+Q$ and $\boldsymbol{e}_{\varphi}$ direction spin, the photon vector potential (80) is similar to vector potential (70), it should not produce $\pi^{+}$meson charge $+Q$, but it produces $-\boldsymbol{e}_{\varphi}$ direction spin. The spin from the vector potentials (79) and (80) are counteracted, which make the $\pi^{+}$meson spin $S=0$. So, the $\pi^{+}$meson has the two kinds of photon vector potentials (79) and (80). Its condensation photon-couple numbers density $\rho_{\pi^{+}}(r, \omega)$ is

$$
\rho_{\pi^{+}}(r, \omega)=g n_{\pi^{+}} \mathrm{e}^{-a_{0} r^{3}} \omega \mathrm{e}^{-b_{0} \omega^{2}},
$$

and the $\pi^{+}$meson mass is

$$
m_{\pi^{+}}=\iint m_{\omega} \rho_{\pi^{+}}(r, \omega) \mathrm{d} V \mathrm{~d} \omega=\frac{2 \pi n_{\pi^{+}} \hbar g}{3 a_{0} b_{0} \sqrt{b_{0}} c^{2}} .
$$

3) The $\pi^{-}$meson structure

The $\pi^{-}$meson has mass and negative charge, and has not spin, it also has two photon vector potentials, they are

$$
\boldsymbol{A}(t)=g(\theta-\omega t) \boldsymbol{A}_{0}=\frac{1}{2 \pi} A_{0} \int_{0}^{2 \pi} \mathrm{e}^{i k(\theta-\omega t)} \mathrm{d} k \boldsymbol{e}_{\theta}
$$

and

$$
\boldsymbol{A}(t)=g(\theta+\omega t) \boldsymbol{A}_{0}=\frac{1}{2 \pi} A_{0} \int_{0}^{2 \pi} \mathrm{e}^{i k(\theta+\omega t)} \mathrm{d} k \boldsymbol{e}_{r} .
$$

The photon vector potential (83) produces $\pi^{-}$meson charge $-Q$ and $-\boldsymbol{e}_{\varphi}$ direction spin, and the photon vector potential (84) only produces $\boldsymbol{e}_{\varphi}$ direction spin, they make the $\pi^{-}$meson spin $S=0$ and charge $-Q$. Its condensation photon-couple numbers density $\rho_{\pi^{-}}(r, \omega)$ is

$$
\rho_{\pi^{-}}(r, \omega)=g n_{\pi^{-}} \mathrm{e}^{-a_{0} r^{3}} \omega \mathrm{e}^{-b_{0} \omega^{2}},
$$

and the $\pi^{-}$meson mass is

$$
m_{\pi^{-}}=\iint m_{\omega} \rho_{\pi^{-}}(r, \omega) \mathrm{d} V \mathrm{~d} \omega=\frac{2 \pi n_{\pi^{-}} \hbar g}{3 a_{0} b_{0} \sqrt{b_{0}} c^{2}},
$$

the ratio of their mass is

$$
m_{\pi^{0}}: m_{\pi^{+}}: m_{\pi^{-}}=n_{\pi^{0}}: n_{\pi^{+}}: n_{\pi^{-}} .
$$




\section{The Dark Particle Structural Theory}

According to the present observations, dark energy and dark matter are two chief constituents of our universe comprising respectively about $69 \%$ and $26 \%$ of its total energy [20], i.e., various cosmological observations indicate that almost $95 \%$ of the energy content of our universe is in some dark form that can only be detected through its gravitational effects. The dark sector involves some kind of cold dark matter (CDM), such as heavy weakly interacting particles or primordial black holes, and dark energy, which could be a manifestation of a cosmological constant $(\Lambda)$ or some exotic matter field [21]. There are many candidates to represent dark matter, such as axion, neutralinos, gravitino and photino, they have light mass, not charge and spin, and don't send observed radiation, they are called dark particle or dark matter.

The dark matter can be composed by one condensation photon-couple, two condensation photon-couple, ..., $n$ condensation photon-couple, their mass are

$$
\left(\frac{2 \hbar \omega}{c^{2}}\right),\left(2 \frac{2 \hbar \omega}{c^{2}}\right),\left(3 \frac{2 \hbar \omega}{c^{2}}\right), \cdots,\left(n \frac{2 \hbar \omega}{c^{2}}\right),
$$

we call this sort of dark matter as darktino.

The dark matter can be constituted by the distribution of condensation photon couples, but there are not the distribution of non-condensable photons. The condensation photon-couple numbers density $\rho_{d}(r, \omega)$ is

$$
\rho_{d}(r, \omega)=g n_{d} \mathrm{e}^{-a_{0} r^{3}} \omega \mathrm{e}^{-b_{0} \omega^{2}}
$$

and the dark particle mass is

$$
m_{d}=\iint m_{\omega} \rho_{d}(r, \omega) \mathrm{d} V \mathrm{~d} \omega=\frac{2 \pi n_{d} \hbar g}{3 a_{0} b_{0} \sqrt{b_{0}} c^{2}} .
$$

We call this sort of dark matter as dark particle, and the darktino and dark particle can further constitute the dark matter. In order to obtain the light mass $m_{d}$, the parameter $n_{d}$ should be small.

\section{Conclusion}

In this paper, we have proposed a new single photon theory, which is the vector potential $\boldsymbol{A}$ rotation at the vertical motion direction of photon, it can produce the microscopic electric field and magnetic field, and they satisfy the Maxwell equations. We have calculated photon spin, momentum, energy, and found there are left-handed and right-handed photon, and they have wave-particle duality in the theory. We have given the relation between photon electric field intensity and energy. In addition, we study the multi-photon theory and the dynamics theory between photons interaction. On this basis, we have further given the compound structures of electron, proton, neutron, neutrino, $\pi$ meson, dark matter with the photon theory, and calculated their mass, charge and spin, all the results should be tested by experiments. 


\section{Acknowledgements}

This work was supported by the Scientific and Technological Development Foundation of Jilin Province (no.20130101031JC).

\section{References}

[1] Planck, M. (1901) Annalen der Physik, 4, 561.

[2] Lenard, P. (1902) Annalen der Physik, 8, 169.

[3] Einstein, A. (1905) Annalen der Physik, 17, 132. https://doi.org/10.1002/andp.19053220607

[4] Compton, A.H. (1923) Physical Review Journals Archive, 22, 409. https://doi.org/10.1103/PhysRev.22.409

[5] Lamb, W.E. (1995) Applied Physics B, 60, 77. https://doi.org/10.1007/BF01135846

[6] Pais, A. (1982) The Science and the Life of Albert Einstein. Oxford, Section 19f, p. 382.

[7] Zavatta, A., Viciani, S. and Bellini, M. (2004) Science, 306, 660. https://doi.org/10.1126/science. 1103190

[8] Jacques, V., Wu, E., Grosshans, F. and Treussart, F. (2008) Physical Review Letters, 100, Article ID: 220402. https://doi.org/10.1103/PhysRevLett.100.220402

[9] Bertocchi, G., Alibart, O. and Ostrowsky, D.B. (2006) Journal of Physics B: Atomic, Molecular and Optical Physics, 39, 1011. https://doi.org/10.1088/0953-4075/39/5/001

[10] Lavoie, J., Donohue, J.M., Wright, L.G., Fedrizzi, A. and Resch, K.J. (2013) Nature Photonics, 7, 363. https://doi.org/10.1038/nphoton.2013.47

[11] Fortsch, M., Furst, J.U., Wittmann, C. and Strekalov, D. (2013) Nature Communications, 4, 1818. https://doi.org/10.1038/ncomms2838

[12] Kuhn, A., Hennrich, M. and Rempe, G. (2002) Physical Review Letters, 89, Article ID: 067901. https://doi.org/10.1103/PhysRevLett.89.067901

[13] Kurtsiefer, C., Mayer, S., Zarda, P. and Weinfurter, H. (2000) Physical Review Letters, 85, 290. https://doi.org/10.1103/PhysRevLett.85.290

[14] Polycarpou, C., Cassemiro, K.N., Venturi, G., Zavatta, A. and Bellini, M. (2012) Physical Review Letters, 109, Article ID: 053602. https://doi.org/10.1103/PhysRevLett.109.053602

[15] Morin, O., Fabre, C. and Laurat, J. (2013) Physical Review Letters, 111, Article ID: 213602. https://doi.org/10.1103/PhysRevLett.111.213602

[16] Qin, Z., Prasad, A., Brannan, T., et al. (2015) Light. Science and Applications, 4, 1.

[17] Sych, D., Rehacek, J., Hradil, Z., et al. (2012) Physical Review A, 86, Article ID: 052123. https://doi.org/10.1103/PhysRevA.86.052123

[18] Bent, N., Qassim, H., Tahir, A., et al. (2015) Physical Review X, 5, Article ID: 041006. https://doi.org/10.1103/PhysRevX.5.041006

[19] Zajonc, A. (2003) Opt and Photon, News Trends, 3, S-2.

[20] Ade, P.A.R., et al. (2016) Astronomy \& Astrophysics, 13, 594.

[21] Kumar, S. and Nunes, R.C. (2016) Physical Review D, 94, Article ID: 123511. https://doi.org/10.1103/PhysRevD.94.123511 